\title{
Descrição do potencial de retenção de nitrogênio em frangas de postura por diferentes metodologias: mínima retenção
}

\author{
Description of the potential for nitrogen retention in pullets by different methodologies: \\ minimum retention
}

\author{
Edney Pereira da Silva ${ }^{I^{*}}$ Nilva Kazue Sakomura ${ }^{\mathrm{I}}$ Melina Aparecida Bonato ${ }^{\mathrm{I}}$ \\ Daniella Carolina Zanardo Donato ${ }^{\mathrm{I}}$ Nelson José Peruzzi ${ }^{\mathrm{I}}$ João Batista Kochenborger Fernandes ${ }^{\mathrm{II}}$
}

RESUMO

Objetivou-se com esta pesquisa descrever o potencial de retenção de nitrogênio por duas técnicas: abate comparativo $e$ balanço de nitrogênio. Também se objetivou comparar os modelos ajustados para cada técnica e estimar as exigências de mantença para frangas de postura. Os ensaios foram realizados no período de 14 a 28, 56 a 70 e 98 a 112 dias de idade, utilizando 168 frangas. Os ensaios tiveram sete tratamentos e oito repetições. Os tratamentos consistiram de níveis de proteina variando de 75 a $435 \mathrm{gkg}^{-1}$ de matéria seca. As variáveis coletadas pelo abate comparativo foram nitrogênio ingerido e depositado e, pela técnica do balanço de nitrogênio, ingestão e excreção de nitrogênio. $O$ intercepto da relação exponencial entre nitrogênio excretado e ingerido foi considerado a exigência de mantença. Os modelos ajustados foram comparados pelo teste da razão de máxima verossimilhança. As técnicas comparadas descreveram de forma diferente a exigencia de mantença de nitrogênio. Para o abate comparativo, a exigência de mantença foi estimada em 342 , 372 e $543 \mathrm{mg} / \mathrm{kg} \mathrm{PC}^{0,67}$ e para o balanço de nitrogênio foi de 342, 225 e $284 \mathrm{mg} /{ }_{\mathrm{kg}} \mathrm{PC}^{0,67}$, para os periodo de 14 a 28, 56 a 70 e 98 a 112 dias de idade, respectivamente.

Palavras-chave: exigência de mantença, balanço de nitrogênio, abate comparativo.

\section{ABSTRACT}

The objectives of this study were to describe the potential for nitrogen retention by two techniques: comparative slaughter and nitrogen balance; compare the adjusted models for each technique and estimate the maintenance requirement for pullets. Assays were performed during 14 to 28, 56-70 and 98 to 112 days of age, using 168 pullets. The design was completely randomized with seven treatments and eight replications. Treatments consisted of protein levels ranging from 75 to $435 \mathrm{gkg}^{-1}$ of dry matter. The variables were collected by comparative slaughter and nitrogen ingested and deposited in nitrogen balance technique intake and nitrogen excretion. The intercept of the exponential relationship between ingested and excreted nitrogen was considered a requirement for maintenance. The adjusted models were compared by using the maximum likelihood ratio. The techniques described differently compared the requirement for maintenance of nitrogen. For comparative slaughter requiring maintenance was estimated at 342, 372 and $543 \mathrm{mg} / \mathrm{kg} P \mathrm{C}^{0,67}$ and for nitrogen balance was 342, 225 and $284 \mathrm{mg} / \mathrm{kg} \mathrm{PC}^{0,67}$ for the period of 14 to $28,56-70$ and 98 to 112 days of age, respectively.

Key words: requirement for maintenance, nitrogen balance, comparative slaughter.

\section{INTRODUÇÃO}

Conforme o modelo de REEDS \& LOBLEY (1980), para o metabolismo proteico (fluxo de proteína do corpo $\mathrm{Q}=$ ingestão $\mathrm{I}+$ degradação $\mathrm{D}=$ síntese $\mathrm{S}+$ oxidação $\mathrm{O}$ ), a síntese de proteína pode ocorrer a partir de um pool de aminoácidos livres, formado por aminoácidos de origem dietética e da degradação da proteína corporal. Uma característica da síntese proteica $(\mathrm{S}=\mathrm{Q}-\mathrm{O})$ pelo modelo de turnover proteico é que: mesmo no equilíbrio de energia ou de nitrogênio, uma quantidade considerável de proteína é sintetizada e contribuirá para o gasto energético do animal (REEDS \& LOBLEY, 1980), ou seja, existe uma mínima retenção de nitrogênio mesmo em estado de equilíbrio de nitrogênio, cuja interpretação nutricional é a exigência de mantença.

A exigência de mantença de nitrogênio vem sendo estudada por várias metodologias, dentre as mais tradicionais tem-se: a técnica do balanço de nitrogênio $(\mathrm{BN})$

\footnotetext{
IFaculdade de Ciências Agrárias e Veterinárias, Universidade Estadual Paulista Jaboticabal (UNESP), 14883-378, Jaboticabal, SP, Brasil E-mail: edneysilva@oi.com.br.*Autor para correspondência.

${ }^{\text {IIC }}$ entro de Aquicultura, UNESP, Jaboticabal, SP, Brasil.
} 
e o abate comparativo (AC). São escassos estudos dessa natureza, sobretudo com frangas de reposição. Nos estudos encontrados, a exigência mínima de nitrogênio (NMR) para compensar as perdas inevitáveis do turnover proteico foi estimada em 760 e $383 \mathrm{mg} /{ }_{\mathrm{kg}} \mathrm{PC}^{0,75} \mathrm{~d}^{-1}$ pelo AC (ALBINOet al., 1994) e BN (BASAGLIAet al., 1998), respectivamente, resultando em uma diferença de $377 \mathrm{mg} / \mathrm{kg}_{\mathrm{g}} \mathrm{PC}^{0,75} \mathrm{~d}^{-1}$ para frangas de reposição da mesma linhagem. As técnicas do BN e AC foram comparadas por FILARDI et al. (2000), os quais estimaram valores $\left(600 \mathrm{e} 326 \mathrm{mg} / \mathrm{kPC}^{0,75} \mathrm{~d}^{-1}\right.$ para o $\mathrm{AC} \mathrm{e} \mathrm{BN}$, respectivamente) que distanciavam as técnicas em $274 \mathrm{mg}$ / ${ }_{\mathrm{kg}} \mathrm{PC}^{0,75} \mathrm{~d}^{-1}$. É possível observar que as diferenças entre AC e BN se mantêm, independente do estudo, e esse resultado pode sustentar a hipótese de que alguma constante fisiológica não vem sendo considerada nos estudos nem nas explicações das diferenças existentes entre as técnicas. Considerando o exposto, Objetivou-se com esta pesquisa descrever o potencial de retenção de nitrogênio por duas técnicas: abate comparativo e balanço de nitrogênio. Também se objetivou comparar os modelos ajustados para cada técnica e estimar as exigências de mantença para frangas de postura.

\section{MATERIAL E MÉTODOS}

Os experimentos foram conduzidos no Laboratório de Ciências Avícolas do Departamento de Zootecnia da Faculdade de Ciências Agrárias e Veterinárias - FCAV/UNESP, Jaboticabal - SP. Foram realizados três ensaios no período de 14 a 28 (ensaio I), 56 a 70 (ensaio II) e 98 a 112 (ensaio III) dias de idade, utilizando 56 frangas Dekalb White ${ }^{\circledR}$, em cada. O delineamento utilizado foi inteiramente ao acaso, com sete tratamentos e oito repetições de uma ave por unidade experimental para controle individual. Os tratamentos consistiram de rações com níveis decrescentes de proteína, variando 435 (N6); 364 (N5); 292 (N4); $220(\mathrm{~N} 3) ; 150(\mathrm{~N} 2)$ e 75 (N1) $\mathrm{gkg}^{-1}$ de matéria seca (MS).

Foram formuladas duas rações para atender o mínimo dos nutrientes e energia metabolizável (EMAn) sugeridos por ROSTAGNO et al. (2005), exceto em proteína. A primeira ração continha $435 \mathrm{gkg}^{-1}$ de proteína, com deficiência relativa de $37 \%$ de Met+Cis digestível (Met+Cis) em relação aos demais. A segunda ração foi formulada para ser isenta de proteína (DIP) e atender aos demais nutrientes e EMAn (Tabela 1). Os níveis experimentais foram obtidos pela técnica da diluição (FISHER \& MORRIS, 1970), diluindo-se a ração N6 sucessivamente com a DIP para obtenção dos níveis intermediários.

Os ensaios foram realizados no galpão de metabolismo dotado de sistema de pressão negativa para controlar a temperatura na zona de termoneutralidade de cada fase. As aves foram alojadas individualmente em gaiolas de metabolismo, equipadas com comedouros individuais e bebedouros tipo nipple. O peso corporal na montagem dos ensaios foram $125 \pm 2 \mathrm{~g}, 449 \pm 14,8 \mathrm{~g}$ e $889 \pm 18 \mathrm{~g}$ nos ensaios I, II e III, respectivamente. Os ensaios duraram 15 dias, o período de coleta de dados e as variáveis coletadas variaram de acordo com a técnica. O período de adaptação consistiu de fornecimento de ração ad libitum para determinar o máximo consumo por quilo de peso metabólico sob as condições experimentais e, em seguida, o fornecimento foi controlado, corrigido para o peso metabólico.

Na técnica do AC, as variáveis coletadas foram nitrogênio ingerido (NI) e nitrogênio depositado (ND) no intervalo de 14 dias. $\mathrm{O}$ fornecimento de ração foi corrigido para o peso metabólico da ave a partir do terceiro dia de experimento, como base no máximo consumo dos dias anteriores. Essa correção foi repetida no quinto e décimo dia de experimento. O NI e ND foram obtidos no período de 14 dias, portanto considerou-se o período de consumo ad libitum.

As deposições de proteína corporal foram determinadas por meio de abates comparativos. Foram abatidas 18 aves no início de cada ensaio e, ao final do ensaio, quatro aves, com peso médio de cada tratamento, foram sacrificadas com o uso $\mathrm{CO}_{2}$, após jejum sólido de 36 horas. As carcaças (carcaça+vísceras+penas+sangue) foram autoclavadas durante seis horas a $130^{\circ} \mathrm{C}$ e $152 \mathrm{kPa}$ de pressão. As amostras foram desengorduradas para posterior análise de nitrogênio pelo método de Kjeldahl (AOAC, 1995) em destilador de nitrogênio (Kjeltec ${ }^{\mathrm{TM}} 8400$; Foss ${ }^{\circledR}$ ). As deposições de proteína $(\mathrm{N} \times 6,25)$ foram obtidas pela diferença entre a quantidade proteína no final e no início de cada ensaio.

Para técnica do balanço de nitrogênio, coletaramse ingestão e excreção de nitrogênio, referente aos últimos 10 dias de experimento. Os ensaios foram divididos em adaptação (cinco dias) e coleta total de excretas (10 dias), conforme sugere os pesquisadores de Göttingen (SAMADI \& LIEBERT, 2006).

Ao final da adaptação, foi iniciado o período de coleta de dados: consumo de ração e coleta total de excretas e, neste dia, as aves foram pesadas para correção do fornecimento de ração, conforme descrito para o AC. As excretas foram coletadas uma vez ao dia e no final de cada ensaio foram pesadas e congeladas $\left(-20^{\circ} \mathrm{C}\right)$ até a présecagem. As amostras foram liofilizadas por 72 horas $\left(-80^{\circ} \mathrm{C} ;-80 \mathrm{kPa}\right)$ e, na sequência, processadas em moinho de bola. Os teores de nitrogênio total das amostras de excretas foram quantificados conforme descrito para AC. O nitrogênio depositado (ND) foi obtido pela diferença entre nitrogênio ingerido e excretado. As variáveis NI e ND foram obtidas diretamente na técnica do $\mathrm{AC}$, enquanto o nitrogênio excretado (NEX) foi obtido pela subtração entre NI e ND, como sendo: NEX =NI-ND. Para o BN, obteve-se ND pela diferença entre NI e NEX, sendo ND = NI-NEX. As 
Tabela 1 - Composição das rações experimentais $\left(\mathrm{gkg}^{-1}\right)$.

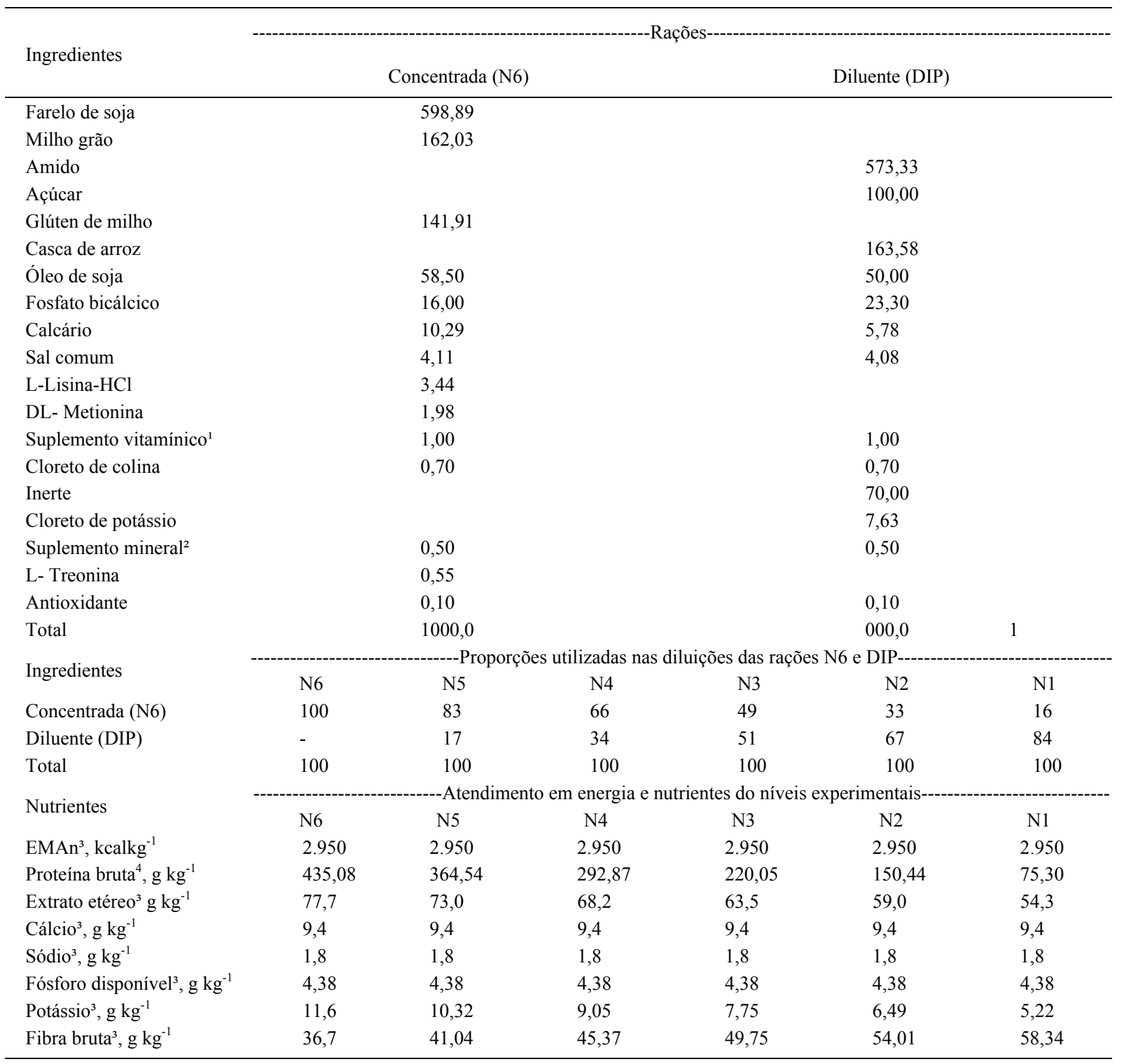

${ }^{1}$ Conteúdo $\mathrm{kg}^{-1}$ : Ácido fólico $=950 \mathrm{mg}$, Ácido pantotênico $=12.000 \mathrm{mg}$, Niacina $=38.000 \mathrm{mg}$, Biotina $=60 \mathrm{mg}$, Vit. A $=8.000 .000 \mathrm{UI}$, Vit. B1 $=2.400 \mathrm{mg}$, Vit. B12 $=12.000 \mathrm{mg}$, Vit. B2 $=5.950 \mathrm{mg}$, Vit. B6 $=2.500 \mathrm{mg}$, Vit. D3 $=2.300 .000 U \mathrm{UI}, \mathrm{Vit} . \mathrm{E}=12.350 \mathrm{mg}$, Vit. K3 $=1.800 \mathrm{mg}$, Se $=300 \mathrm{mg}$, antioxidante $=250 \mathrm{mg}$, Veículo q.s.p. $1.000 \mathrm{~g}$. ${ }^{2}$ Conteúdo $\mathrm{kg}^{-1}: \mathrm{Mn}=200.000 \mathrm{mg}, \mathrm{Fe}=100.000 \mathrm{mg}, \mathrm{Zn}=160.000 \mathrm{mg}, \mathrm{Cu}=$ $16.000 \mathrm{mg}$, I $=1.500 \mathrm{mg}$, Veículo q.s.p. $1.000 \mathrm{~g} ;{ }^{3}$ Valores expressos com base na matéria natural; ${ }^{4}$ Valores expressos com base na matéria seca $(\mathrm{MS}) \mathrm{N} 6=867,7 ; \mathrm{N} 5=860,9 ; \mathrm{N} 4=854,0 ; \mathrm{N} 3=847,2 ; \mathrm{N} 2=840,8$ e N1 $=834,0 \mathrm{gkg}^{-1} \mathrm{de} \mathrm{MS}_{\text {. }}$

respostas de NEX e NI de cada fase foram ajustadas pela equação 1:

$\mathrm{NEX}=\mathrm{NMR} \times e^{(\mathrm{b} \times \mathrm{NI})}+\varepsilon$, sendo NMR e $\mathrm{b}>0 \quad$ Eq. [1]

Em que NEX é nitrogênio excretado, mg/ ${ }_{\mathrm{kg}} \mathrm{PC}^{0,67} \mathrm{~d}^{-1}$; NI é nitrogênio ingerido; NMR é intercepto da função, obtido quando $\mathrm{NI}=0$, ou seja, as perdas inevitáveis de nitrogênio, cuja interpretação nutricional é a exigência de mantença de nitrogênio; b é taxa de crescimento da função e $e$ é o número de Euler; $\varepsilon$ é erro aleatório atendendo as pressuposições de normalidade. Os modelos obtidos para cada técnica foram submetidos à análise de identidade de modelo e igualdade de parâmetros pelo teste da razão de verossimilhança, utilizando-se a estatística qui-quadrado $\chi^{2}$, conforme REGAZZI (2003).

As hipóteses iniciais admitidas são: A) - $\mathrm{H}_{0}$ : as equações são idênticas para ambas as técnicas, ou seja, uma equação comum pode ser usada como estimativa das equações envolvidas e B) - $\mathrm{H}_{0}$ : um determinado subconjunto 
de parâmetros é igual nas técnicas. Para verificar a identidade de cada modelo e igualdade de parâmetros estimados de acordo com a técnica, foram adotadas as hipóteses generalizadas para modelo com dois parâmetros.

$\mathrm{H}_{0}^{1}: \mathrm{a}_{1}=\ldots=\mathrm{a}_{\mathrm{g}}(=\mathrm{a})$ vs $\mathrm{H}_{\mathrm{a}}^{1}$ : nem todos $\mathrm{a}_{1}$ são iguais; $\mathrm{H}_{0}^{2}: \mathrm{b}_{1}=\ldots=\mathrm{b}_{\mathrm{g}}(=\mathrm{b})$ vs $\mathrm{H}_{\mathrm{a}}^{2}$ : nem todos $\mathrm{b}_{1}$ são iguais; $\mathrm{H}_{0}{ }^{3}: \mathrm{a}_{1}=\ldots=\mathrm{a}_{\mathrm{g}}(=\mathrm{a})$ e $\mathrm{b}_{1}=\ldots=\mathrm{b}_{\mathrm{g}}(=\mathrm{b}) v s \mathrm{H}_{\mathrm{a}}^{3}$ : pelo menos uma igualdade e uma desigualdade; em $\mathrm{g}$, significa parâmetro generalizado para ambas as técnicas.

Os modelos foram parametrizados conjuntamente com auxílio da inclusão de duas variáveis "dummy", conforme o modelo generalizado. $D_{i}=1$ se a observação pertence à técnica $i$ e 0 em caso contrário; $i=$ técnica AC e BN. Os coeficientes foram estimados conforme a seguinte descrição:

$\mathrm{NEX}=D_{1}\left[\mathrm{NMR} \times e^{(\mathrm{b} \times \mathrm{NN})}\right]+D_{2}\left[\mathrm{NMR} \times e^{(\mathrm{b} \times \mathrm{NN})}\right]+\varepsilon \quad$ Eq.[2]

Os dados foram ajustados conforme a Eq. [2], utilizando as hipóteses generalizadas para obtenção de subconjuntos paramétricos. Considerando $\theta$ a representação de todos os parâmetros $\left(a_{1} \ldots a_{g} \ldots, b_{1} \ldots b_{g} e\right.$ $\sigma^{2}$ ), admitiu-se a hipótese de nulidade $\mathrm{H}_{0}: \theta \in \omega v s \mathrm{H}_{\mathrm{z}}: \theta \in \omega^{\mathrm{\varepsilon}}$, em que $\omega$ é um subconjunto do espaço paramétrico $\Omega$ e $\omega^{\mathrm{c}}$ é o complemento de $\omega, \operatorname{com} \Omega=\omega \cup \omega^{c}$. Foi ajustado o modelo completo $\Omega$ e obteve-se ${ }^{\widehat{\sigma}_{\Omega}^{2}}=\left(\frac{\mathrm{SQR}_{\Omega}}{\mathbf{n}}\right)$, em que $S Q R_{\Omega}$ é a soma do quadrado do resíduo para $\Omega$; e $\mathbf{p}_{\Omega}$ é o número de parâmetros em estimados em $\Omega$. Em seguida, ajustou-se o modelo reduzido em $\omega$, conforme as hipóteses $\mathrm{H}_{0}$ e obteve-se $\widehat{\sigma}_{\omega}^{2}=\left(\frac{\mathrm{SQR}_{\omega}}{\mathbf{n}}\right)$, em que $\mathrm{SQR}_{\omega}$ é a soma do quadrado do resíduo para $\omega$; e $\mathbf{P}_{\omega}$ é o número de parâmetros em estimados em $\omega$. O teste da razão de verossimilhança (L) foi calculado da seguinte forma: $L=\left(\frac{\hat{\sigma}_{\Omega}^{2}}{\hat{\sigma}_{\omega}^{2}}\right)^{n / 2}$ e comparado pela

estatística $\chi^{2}$ considerando $\alpha=0,05$. Os dados foram analisados usando pacote estatístico SAS, versão 9.1, por meio do procedimento NLIN para ajuste de modelos.

\section{RESULTADOS E DISCUSSÃO}

Os resultados obtidos para NI e NEX estão apresentados na tabela 2. A relação entre NI e NEX apresenta coerência fisiológica, além de oferecer sustentação estatística para extrapolação da respostas de excreção à ingestão de nitrogênio. A trajetória do NEX foi modelada pela função exponencial e mostra que a interceptação ou NMR, no eixo $\mathrm{NEX}$, para $\mathrm{NI}=0$ é uma distância próxima dos dados observados, que confere robustez ao procedimento (Tabela 3).

Portanto, a metodologia aplicada oferece uma boa aproximação ao objeto de estudo. O NMRé o indicativo indireto da degradação proteica para permitir a síntese mínima exigida pelo turnover em condições de alimentação livre de nitrogênio, obtida com aves recebendo nitrogênio na dieta. Na privação de ingestão de proteína, parte da proteína corporal é degradada para compor o pool de aminoácidos livres e, em seguida, uma parte dos aminoácidos mobilizados é re-sintetizado para formar uma nova proteína de prioridade metabólica (REEDS \& LOBLEY, 1980). Acredita-se que os resíduos da oxidação dos aminoácidos foram contabilizados na excreção das aves, quando extrapola-se o NEX para NI=0.

As informações práticas extraídas das taxas ou valores de $\mathrm{b}$ desta função estão associadas à retenção de nitrogênio, logo, quanto maior a deposição de nitrogênio ou proteína, menor é a taxa de excreção e mais suavizado é a trajetória da função. Da mesma forma, quando se aproxima do limite de deposição, a taxa de excreção aumenta, uma vez que a deposição de nitrogênio é limitada pelo potencial genético. Esse parâmetro não será enfatizado neste estudo. Para maiores detalhes ver Silva et al. (2013).

A comparação dos coeficientes (NMR e b) dos modelos ajustados para cada técnica (AC e BN) demonstrou que a variância obtida com o modelo irrestrito $(\Omega)$ não diferiu do modelo restrito em GD $\mathrm{G}^{1}$ para fase 14-28 dias. Desse modo, aceita-se a hipótese $\mathrm{H}_{0}^{3}$ em que se verificou uma igualdade e uma desigualdade, portanto o parâmetro de interesse (NMR) do modelo pode ser generalizado para ambas as técnicas nessa fase, logo, a exigência de mantença da ave foi estimada em $342 \mathrm{mg} / \mathrm{kg}^{0} \mathrm{PC}^{0,67} \mathrm{~d}^{-1}$. Apesar de apresentar igualdade em pelo menos um parâmetro, os modelos obtidos não têm a mesma identidade, ou seja, as taxas (b) de respostas são específicas para cada técnica, portanto, um único modelo não é capaz de explicar as respostas obtidas.

Nas demais fases (56 a 70 e 96-112 dias), a estatística aplicada mostrou que um subconjunto do espaço paramétrico $(G)$ não representa o espaço paramétrico $(\Omega)$, rejeitando a hipótese de nulidade com valor de $\mathrm{P}<0,001$; logo, conclui-se que as técnicas descreveram, de forma diferente, a NMR. As maiores variâncias foram obtidas para hipótese $\mathrm{H}_{0}^{\mathrm{G} 1}$ : $\mathrm{MNR}_{\mathrm{BN}}=\mathrm{NMR}_{\mathrm{AC}}=\mathrm{NMR}$. Desse modo, a exigência de mantença pelo $\mathrm{BN}$ foi estimada em $225 \mathrm{e}$ $284 \mathrm{mg} / \mathrm{kg} \mathrm{PC}^{0,67} \mathrm{~d}^{-1}$ para fase de cria e recria, respectivamente. Para o AC, os valores foram de 372 e $543 \mathrm{mg} /{ }_{\mathrm{kg}} \mathrm{PC}^{0,67} \mathrm{~d}^{-1}$, considerando as mesma fases.

Este resultado sustenta a hipótese de que a composição do NEX para o AC pode conter um fator não considerado pelo BN, além da oxidação dos aminoácidos. Em ensaios de BN com galos, LEVEILLE et al. (1960) comprovaram a influência das perdas de penas sobre a exigência de mantença. Esses autores verificaram que as determinações isoladas dos aminoácidos se correlacionavam altamente $(\mathrm{r}=0,86 \mathrm{P}<0,01)$ com a composição aminoacídica da pena.

$\mathrm{Na}$ tentativa de elucidar este efeito da pena no presente trabalho, utilizou-se o coeficiente $0,04 \%$ $\mathrm{dia}^{-1}$ de perdas de proteína (ou nitrogênio/6,25) via penas, 
Tabela 2 - Nitrogênio ingerido (NI mg/kg $\mathrm{PC}^{0,67} \mathrm{~d}^{-1}$ ) e nitrogênio excretado $\left(\mathrm{NEX} \mathrm{mg/kg} \mathrm{PC}^{0,67} \mathrm{~d}^{-1}\right.$ ) de frangas de postura submetidas níveis crescente de nitrogênio, obtidos pela técnica do abate comparativo e balanço de nitrogênio ${ }^{1}$

\begin{tabular}{|c|c|c|c|c|c|c|c|}
\hline \multirow{2}{*}{ Variáveis } & \multicolumn{7}{|c|}{--Níveis de nitrogênio----- } \\
\hline & N1 & $\mathrm{N} 2$ & $\mathrm{~N} 3$ & $\mathrm{~N} 4$ & N5 & N6 & N7 \\
\hline \multicolumn{8}{|c|}{ 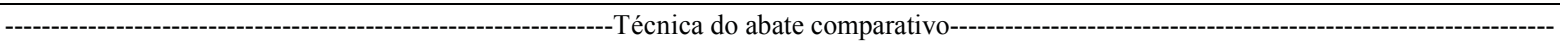 } \\
\hline NI & $565 \pm 16$ & $1588 \pm 262$ & $2309 \pm 56$ & $3043 \pm 75$ & $3884 \pm 93$ & $4317 \pm 94$ & $798 \pm 29$ \\
\hline \multirow[t]{2}{*}{ NEX } & $342 \pm 49$ & $668 \pm 29$ & $1164 \pm 38$ & $1748 \pm 67$ & $2661 \pm 92$ & $3143 \pm 72$ & $194 \pm 40$ \\
\hline & \multicolumn{4}{|c|}{ 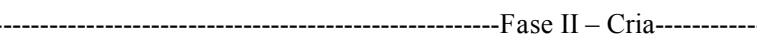 } & \multirow{3}{*}{$\begin{array}{l}2850 \pm 36 \\
2091 \pm 30\end{array}$} & - r & ------------ \\
\hline \multirow{3}{*}{$\begin{array}{l}\text { NI } \\
\text { NEX }\end{array}$} & \multirow{2}{*}{$\begin{array}{l}597 \pm 11 \\
430 \pm 18\end{array}$} & $1267 \pm 7$ & \multirow{2}{*}{$\begin{array}{l}1721 \pm 14 \\
967 \pm 30\end{array}$} & $2432 \pm 72$ & & \multirow{2}{*}{$\begin{array}{l}3431 \pm 26 \\
2698 \pm 33\end{array}$} & $614 \pm 4$ \\
\hline & & $731 \pm 35$ & & $1681 \pm 76$ & & & $206 \pm 29$ \\
\hline & & & \multicolumn{2}{|c|}{-------Fase III - Recria-------- } & \multirow[b]{2}{*}{$2992 \pm 37$} & \multirow[b]{2}{*}{$3703 \pm 37$} & ---------- \\
\hline \multirow{2}{*}{$\begin{array}{l}\text { NI } \\
\text { NEX }\end{array}$} & $514 \pm 8$ & $1252 \pm 7$ & $1745 \pm 24$ & $2388 \pm 24$ & & & $613 \pm 7,2$ \\
\hline & $421 \pm 24$ & $918 \pm 29$ & \multicolumn{2}{|c|}{$1357 \pm 21$} & $2574 \pm 45$ & $3270 \pm 41$ & $424 \pm 26$ \\
\hline \multicolumn{8}{|c|}{ } \\
\hline NI & \multirow{2}{*}{$\begin{array}{l}589 \pm 13 \\
278 \pm 25\end{array}$} & $1586 \pm 26$ & \multirow{2}{*}{$\begin{array}{l}2285 \pm 48 \\
1017 \pm 60\end{array}$} & $2975 \pm 73$ & \multirow{2}{*}{$\begin{array}{l}3632 \pm 103 \\
2171 \pm 128\end{array}$} & \multirow{2}{*}{$\begin{array}{l}4049 \pm 117 \\
2553 \pm 181\end{array}$} & \multirow{2}{*}{$\begin{array}{l}809 \pm 28 \\
283 \pm 20\end{array}$} \\
\hline \multirow[t]{2}{*}{ NEX } & & $662 \pm 43$ & & $1554 \pm 70$ & & & \\
\hline & & & \multicolumn{2}{|c|}{---------Fase II - Cria---------- } & - & \multirow[b]{2}{*}{$3348,0 \pm 32$} & \multirow[b]{2}{*}{$600,5 \pm 24$} \\
\hline \multirow{2}{*}{$\begin{array}{l}\text { NI } \\
\text { NEX }\end{array}$} & $596 \pm 9$ & $1221,1 \pm 8,5$ & $1706,1 \pm 20$ & $2392,3 \pm 73$ & $2796,2 \pm 37$ & & \\
\hline & $374 \pm 22$ & $572 \pm 20,4$ & $631 \pm 32$ & $1071 \pm 74$ & $1556 \pm 103$ & $2134 \pm 29$ & $261 \pm 15$ \\
\hline \multirow[b]{2}{*}{ NI } & & & \multicolumn{2}{|c|}{----Fase III - Recria----- } & & & \\
\hline & $598 \pm 8$ & $1314 \pm 11$ & $1821 \pm 25$ & $2472 \pm 28$ & $3079 \pm 38$ & $3820 \pm 35$ & $666 \pm 8,8$ \\
\hline NEX & $381 \pm 26$ & $602 \pm 50$ & $763 \pm 56$ & $1350 \pm 72$ & $1613 \pm 104$ & $2646 \pm 59$ & $248 \pm 20$ \\
\hline
\end{tabular}

${ }^{1}$ Média \pm erro; N7, tratamento com teor de proteína bruta similar ao N1 e com concentração de metionina+cistina similar ao N2, obtida da seguinte forma: $\mathrm{N} 7=\mathrm{N} 1+(\mathrm{N} 2-\mathrm{N} 1)$.

determinado por SILVA (2012). Com esse coeficiente, foi possível estimar a perda de penas para cada fase em 93 (inicial), 59 (cria) e 25 (recria) mg de nitrogênio ${ }_{\mathrm{kg}} \mathrm{PC}^{0,67}$. Os valores de nitrogênio perdido via penas foram somados aos NMR das fases inicial, cria e recria, respectivamente, que resultou em uma média de $333 \mathrm{mg} /{ }_{\mathrm{kg}} \mathrm{PC}^{0,67}$; com isso, a diferença entre as técnicas foi minimizada, passando de 1,56:1, sem correção das perdas de nitrogênio via penas, para 1,28:1 (472/333), com a correção.

Em adição, SILVA (2012), baseado em dados não publicados, coletado na FCVA/Unesp-Jaboticabal em ensaio de BN com frangos de corte Cobb (lote misto) de 26 a 37 dias de idade, quantificaram as perdas de penas (PP) de 0,4;0,44; 0,59; 0,61; 0,77 e 0,80g com 10, 20,30,40, 50 e 60g $\mathrm{kg}^{-1}$ de nitrogênio $(\mathrm{N})$ na dieta, respectivamente. Uma relação linear estabelecida entre PP e N $(\mathrm{PP}=0,0086 \times \mathrm{N}+0,3007)$ revelou que o aumento na concentração e consequentemente e ingestão de nitrogênio aumentou a perda de penas. Com base nessa relação, o aumento da ingestão de nitrogênio estimula a renovação e consequentes perdas de penas. Portanto, os valores de NEX obtidos pelo AC, estimado pela diferença entre ingestão e deposição de nitrogênio contêm implicitamente os referidos efeitos.

Para comparar os resultados obtidos neste estudo com os apresentados na literatura, faz-se necessário esclarecer algumas diferenças metodológicas. A exigência de mantença pelo método tradicional é obtida por meio da relação entre a ND e NI, com níveis de proteína dietética suficientemente baixa, para obter valores negativos de ND e assim estabelecer a relação linear entre as variáveis $\mathrm{ND}=$ $-\mathrm{a}+\mathrm{b} \times$ NI. Dessa forma, as perdas inevitáveis, representadas pelo intercepto da função (a), são obtidas pela extrapolação para $\mathrm{NI}=0, \operatorname{logo}, \mathrm{ND}=-\mathrm{a}$, que tende a ser um valor negativo. A diferença entre a metodologia tradicional e atual abordagem situa-se em considerar as perdas inevitáveis. Pela metodologia tradicional, a ingestão para mantença é obtida igualando $\mathrm{ND}=0,(0=-\mathrm{a}+\mathrm{b} \times \mathrm{NI}), \log \mathrm{NI}=\mathrm{a} / \mathrm{b}$, dessa forma, o intercepto (a) é dividido pela inclinação (b), assim, os valores obtidos pela metodologia tradicional tendem a ser diferentes na origem da estimativa. Para evitar confusão entre as metodologias, neste trabalho, a mínima retenção de nitrogênio foi comparada com as perdas inevitáveis (a) estimadas pelo método tradicional.

Os valores médios obtidos em função da técnica, sem considerar a idade, foi 427 e $273 \mathrm{mg} /{ }_{\mathrm{kg}} \mathrm{PC}^{0,67} \mathrm{~d}^{-1}$ para o $\mathrm{AC}$ e $\mathrm{BN}$, respectivamente. As perdas determinadas pelo AC mostraram uma equivalência de 1.56:1 (472/273) ao valor determinado com BN. De forma similar ao obtido nesta pesquisa, FILARDI et al. (2000) encontraram uma equivalência de 1.82:1. As diferenças apresentadas nos 
Tabela 3 - Relação entre nitrogênio excretado (NEX) e ingerido (NI), estimativas dos parâmetros NMR e b do modelo exponencial irrestrito $(\Omega)$ e restrito $(\boldsymbol{\omega})$.

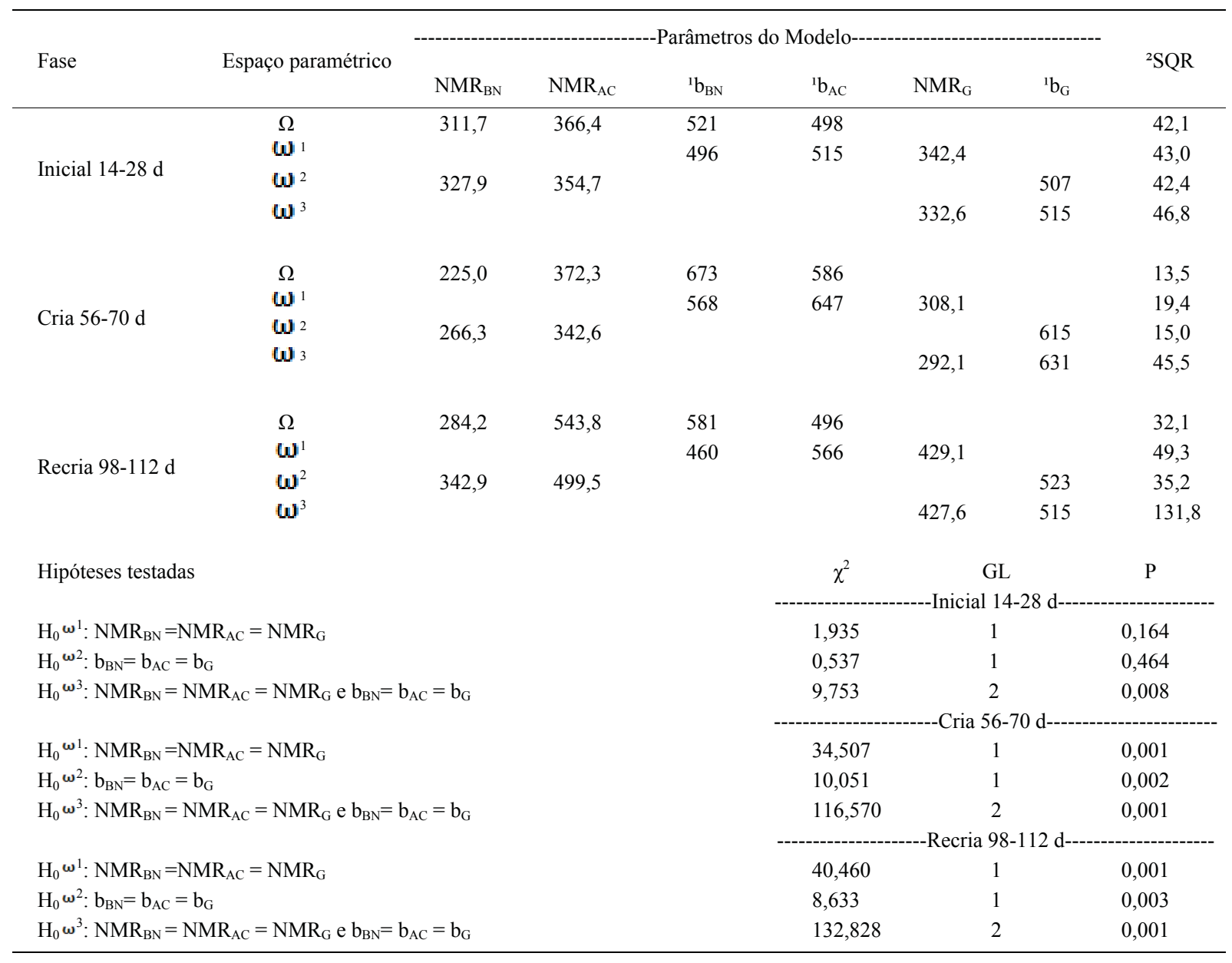

$\mathrm{NEX}=\mathrm{NMR} \times e^{\mathrm{b} \times \mathrm{NI}} ;$ mínima retenção de nitrogênio; $\mathrm{b}$ é taxa de crescimento da função; $e$, o número de Euler

$\mathrm{BN}$, técnica do balanço de nitrogênio; AC, técnica do abate comparativo; G, geral; SQR, variância; $\chi^{2}$, qui-quadrado; GL, grau de liberdade; $\mathrm{P}$, valor de probabilidade; ${ }^{1}$ Valores expressos em potência de $10^{-6} ;{ }^{2}$ Valores expressos em potência de $10^{5}$

$\mathrm{H}_{0} \omega^{1}$ : Os coeficientes NMR são similares, independente da técnica; e $\mathrm{H}_{1}{ }^{\omega 1}$ Os coeficientes NMR são diferentes, dependente da técnica;

$\mathrm{H}_{0} \omega^{2}$ : Os coeficientes b são similares, independente da técnica; e $\mathrm{H}_{1} \omega^{1}$ Os coeficientes b são diferentes, dependente da técnica;

$\mathrm{H}_{0}{ }^{\omega^{3}}$ : Os coeficientes NMR e b são similares, independente da técnica; e $\mathrm{H}_{1}{ }^{\omega^{1}}$ nem todos os NMR e b são iguais, pelo menos uma igualdade e uma desigualdade entre as técnicas;

$\Omega$ é o espaço paramétrico, estimado sem restrição; $\boldsymbol{\omega}$ é um subconjunto do espaço paramétrico $\Omega$ com restrição em um ou mais parâmetros do modelo.

valores obtidos pelo $\mathrm{AC}$ e $\mathrm{BN}$ podem ser atribuídas à fidelidade ao próprio conceito de mantença. Acredita-se que a perda de nitrogênio via renovação de penas não seja contabilizada nos valores de NEX obtidos pela técnica do BN.

Valores similares aos determinados são encontrados na literatura. O NMR encontrado por ALBINO et al. (1994) foi de $377 \mathrm{mg} / \mathrm{kP}^{0,67}\left(410 \mathrm{mg} /{ }_{\mathrm{kg}} \mathrm{PC}^{0,75} \times 0,92\right)$, estimada pelo AC com aves Lohmann. BASAGLIA et al. (1998) encontraram que as perdas foram equivalentes a $236 \mathrm{mg}_{\mathrm{kg}} \mathrm{PC}^{0,67}\left(252,7 \mathrm{mg} / \mathrm{kg}^{0,75} \times 0,92\right)$ pelo BN para aves de mesma linhagem. O NMR é singular a cada genótipo, no entanto, asdiferençasencontradascomDekalbde +17 (Albino et al., 1994) e +37 (BASAGLIAet al., 1998) parecem ser mínimas em relação aos valores obtidos com poedeiras leves da linhagem Lohmann, sobretudo em relação aos valores gerados pelo AC. Similaridade entre genótipos foi encontrada com frangos de corte Cobb (SAMADI\&LIEBERT, 2006) e Ross (SAMADI\&LIEBERT, 2008), que diferenciaram em $\pm 31 \mathrm{mg} / \mathrm{kg}^{0} \mathrm{PC}^{0,67}$.

A metodologia utilizada na determinação influencia a magnitude do NMR. Os maiores valores de perdas são encontrados em estudos com aves em jejum por 48h. Dessa forma, SILVA et al. (2011) encontraram 
$1.067 \mathrm{mg} / \mathrm{kg}^{0} \mathrm{PC}^{0,67}$. Portanto, no estado de jejum prolongado, a degradação da proteína corporal mantém o pool de aminoácidos livres que são direcionados ao fígado, quando sofrem oxidação completa ou podem ser convertidos em glicose ou corpos cetônicos (SILVAet al., 2011). Esses artifícios metabólicos são diferentes daqueles animais criados em regime ad libitum. Por outro lado, a utilização de DIP tende a subestimar os valores de NMR. LEVEILLE \& FISHER (1958) encontraram 99mg/ $/ \mathrm{PC}^{0,67}$ com galos. Portanto, os resultados obtidos são intermediários aos apresentados na literatura. A metodologia aplicada permite simular o estado fisiológico da ave em condições de alimentação livre de nitrogênio, obtida com aves recebendo nitrogênio na dieta, evitando mecanismos indesejados.

\section{CONCLUSÃO}

As técnicas comparadas descreveram de forma diferente a exigência de mantença de nitrogênio. Para o abate comparativo, a exigência de mantença foi estimada em 342,372 e $543 \mathrm{mg} /{ }_{\mathrm{kg}} \mathrm{PC}^{0,67}$ e, para o balanço de nitrogênio, foi de 342, 225 e $284 \mathrm{mg} /{ }_{\mathrm{kg}} \mathrm{PC}^{0,67}$ para o período de 14 a 28,56 a 70 e 98 a 112 dias de idade, respectivamente.

\section{AGRADECIMENTOS}

Aos Pesquisadores Frank Liebert e Angela Sünder do Instituto de fisiologia animal e nutrição animal da Universidade de Georg-August, Goettingen - Alemanha, pela valiosa contribuição. Ao Conselho Nacional de Desenvolvimento Científico e Tecnológico $(\mathrm{CNPq})$ e à Fundação de Amparo a Pesquisa do Estado de São Paulo (FAPESP), pelo apoio financeiro e concessão de bolsas de estudo.

\section{COMITÊ DE ÉTICA E BIOSSEGURANÇA}

Protocolo no 007125-08.

\section{REFERÊNCIAS}

ALBINO, L.F.T. et al. Estimativas das exigências de energia e proteína para frangas de postura em recria. Pesquisa Agropecuaria Brasileira, v.29, n.10, p.1625-1629, 1994.Disponível em: < http://seer.sct.embrapa. br/index.php/pab/article/viewFile/4216/1507>. Acesso em: 22 jun. 2011.

AOAC (ASSOCIATION OF OFFICIAL ANALYTICAL CHEMISTS). Official methods of analysis. Washington, D.C, 1995. 1094p

BASAGLIA, R. et al. Exigências de proteína para frangas de postura de 1 a 18 semanas de idade.Revista Brasileira de Zootecnia, v.27, n.3, p.556-563, 1998. Disponivel em: < http://www.sbz.org.br/ revista/artigos/1970.pdf>. Acesso em: 22 jun. 2011.

FILARDI, R.S. et al.Equações de predição das exigências de proteína bruta para matrizes pesadas em crescimento.Revista Brasileira de Zootecnia, v.29, n.6, p.2308-2315, 2000. Disponivel em: <http:// dx.doi.org/10.1590/S1516-35982004000400010>. Acesso em: 22 jun. 2011.doi:10.1590/S1516-35982004000400010.
FISHER, C.; MORRIS, T.R.The determination of the methionine requirement of laying pullets by a diet dilution technique.British Poultry Science, v.11, n.1, p.67-82, 1970. Disponível em: <http:// dx.doi.org/10.1080/00071667008415793>.Acesso em: 22 jun. 2011. doi:10.1080/00071667008415793.

LEVEILLE, G.A. et al. Amino acid requirements for maintenance in the adult rooster: IV. The requirements for methionine, cystine, phenylalanine, tyrosine and tryptophan; the adequacy of the determined requirements. Journal Nutrition, v.72, n.1, p.8-15, 1960. Disponivel em: <http://jn.nutrition.org/content/72/1/8.full. pdf $>$. Acesso em: 22 jun. 2011.

LEVEILLE, G.A.; FISHER, H. The amino acid requirements for maintenance in the adult rooster: I. Nitrogen and energy requirements in normal and protein-depleted animals receiving whole egg protein and amino acid diets. Journal Nutrition, v.66, n.3, p.441-453, 1958.Disponivel em: <http://jn.nutrition.org/ content/66/3/441.long >. Acesso em: 22 jun. 2011.

REEDS, P.J.; LOBLEY, G.E. Protein synthesis: are there real species differences? Proceedings of the Nutrition Society, v.39, n.1, p.43-52, 1980. Disponível em: <http://dx.doi.org/10.1079/PNS19800007>. Acessoem:26 nov.2011. doi: 10.1079/PNS19800007.

REGAZZI, A.J. Teste para verificar a igualdade de parâmetros e a identidade de modelos de regressão não-linear. Revista Ceres, v.50, n.287, p.9-26, 2003.Disponível em: <http://www.ceres.ufv. br/ceres/revistas/v50n287p19303.pdf >. Acesso em: 23 nov. 2011.

ROSTAGNO, H.S. et al. Tabelas brasileiras para aves e suínos composição de alimentos e exigências nutricionais.Viçosa, MG: UFV, DZO, 2005. 186p.

SAMADI, ; LIEBERT, F. Estimation of nitrogen maintenance requirements and potential for nitrogen deposition in fastgrowing chickens depending on age and sex. Poultry Science, v.85, n.8, p.1421-1429, 2006. Disponível em: <http://ps.fass.org/ content/85/8/1421.full.pdf + html $>$.Acesso em: 22 jun. 2011.

SAMADI; LIEBERT, F. Modelling the optimal lysine to threonine ratio in growing chickens depending on age and efficiency of dietary amino acid utilisation. British Poultry Science, v.49, n.1, p.45-54, 2008.Disponível em: < http://dx.doi. org/10.1080/00071660701821667>. Acesso em: 22 jun. 2011. doi:10.1080/0007166070182166.

SILVA, E.P.Modelos de crescimento e das respostas de frangas de postura submetidas a diferentes ingestões de aminoácidos sulfurados. 2012. 207f. Tese (Doutorado em Zootecnia) -Universidade Estadual Paulista "Julio de Mesquita Filho" SP.

SILVA, E.P. et al. Efeito da idade sobre as perdas endógenas e metabólicas de frangos de corte industrial e caipira. Ciencia Animal Brasileira, v.12, n.1, p.34-47, 2011.Disponível em: <http:// dx.doi.org/10.5216/cab.v12i1.5096>. Acesso em: 21ago. 2012. doi:10.5216/cab.v12i1.5096.

SILVA, E.P et al. Descrição do potencial de retenção de nitrogênio em frangas de postura por diferentes metodologias: máxima deposição e estimativas da ingestão de metionina+cistina. Ciência Rural, v.43, n.11, p.20702077, 2013. Disponível em <http://dx.doi.org/10.1590/ S0103-84782013001100024>. Acesso 10nov.. doi:10.1590/ S0103-84782013001100024. 